\title{
Effectiveness and safety of four tract dilation methods of percutaneous nephrolithotomy: A meta-analysis
}

\author{
YUE WU, YANG XUN, YUCHAO LU, HENGLONG HU, BAOLONG QIN and SHAOGANG WANG \\ Department of Urology, Tongji Hospital, Tongji Medical College, \\ Huazhong University of Science and Technology, Wuhan, Hubei 430030, P.R. China
}

Received December 3, 2018; Accepted January 6, 2020

DOI: $10.3892 /$ etm.2020.8486

\begin{abstract}
Percutaneous nephrolithotomy (PCNL) has become a routine surgical procedure for treating patients with large kidney stones; the fundamental step in this process is the creation of the nephrostomy tract. In the present study, a meta-analysis was performed to compare the effectiveness and safety of different tract dilation techniques for PCNL. Databases were searched from inception to 1 April 2019 to identify relevant randomized controlled trials. The X-ray exposure time, hemoglobin decrease, stone-free rate, transfusion rate, hospital stay and the complication rate associated with the various techniques were analyzed. A total of 11 studies comprising 1,415 cases were enrolled in the meta-analysis. Significant differences in X-ray exposure time [weighted mean difference (WMD), 30.67; 95\% confidence interval (CI), 20.08-41.26; $\mathrm{P}<0.001]$ and hemoglobin decrease (WMD, 0.19; 95\% CI, 0.15-0.23; $\mathrm{P}<0.001)$ were identified between metal telescopic dilation (MTD) and one-shot dilation (OSD). A significantly lower hemoglobin decrease was observed in the balloon dilation (BD) vs. fascial Amplatz dilation (AD) group [WMD, -0.65; 95\%CI, -(0.77-0.52); $\mathrm{P}<0.001$ ]. The transfusion rate was similar between these techniques. The MTD had an obviously higher successful dilation rate compared with that of the OSD, but no significant differences in stone-free rate and transfusion rate were obtained. The present study determined that, compared with other methods, OSD was safer in almost every adult patient, including those that had previously under-
\end{abstract}

Correspondence to: Professor Shaogang Wang, Department of Urology, Tongji Hospital, Tongji Medical College, Huazhong University of Science and Technology, 1095 Jiefang Avenue, Wuhan, Hubei 430030, P.R. China

E-mail: sgwangtjm@163.com

Abbreviations: PCNL, percutaneous nephrolithotomy; RCT, randomized controlled trial; $\mathrm{CI}$, confidence interval; WMD, weighted mean difference; $\mathrm{RR}$, risk ratio; $\mathrm{OR}$, odds ratio; $\mathrm{AD}$, fascial Amplatz dilation; MTD, metal telescopic Alken type dilation; $\mathrm{BD}$, balloon dilation; OSD, one-shot dilation

Key words: percutaneous nephrolithotomy, tract dilation, kidney stones, meta-analysis gone renal surgery; though it is recommended that this should be performed by experienced surgeons. BD was reported to be effective and safer in patients without a history of renal surgery compared to other methods. The present study proposed AD and MTD as safer methods of dilation for patients who have previously undergone kidney surgery.

\section{Introduction}

Among the developments in surgical endourology techniques in the past three decades, percutaneous nephrolithotomy (PCNL) has become a standard treatment strategy with minimal invasiveness for the treatment of large renal stones $(1,2)$. Conventionally, creating the nephrostomy tract is a fundamental process in this technique. At present, there are four major dilation methods for PCNL: Fascial Amplatz dilation (AD), metal telescopic Alken type dilation (MTD), balloon dilation (BD) and one-shot dilation (OSD). BD is generally considered as the most modern and safest technique. It has advantages of reduced complication rates and shorter durations of X-ray exposure (3-5), but its application is limited due to high cost. AD and MTD are inexpensive, but longer durations of application and X-ray exposure are required. The OSD technique, which was first proposed by Frattini et al (6), may achieve the same effects compared with the other three dilation methods (7-13); however, OSD may cause parenchymal damage (14). A previous meta-analysis compared the four dilation methods (15), but only four randomized clinical trials (RCTs) were included and three combinations of tract dilation methods were analyzed. Therefore, the most effective method for the selection of tract dilation remains controversial; surgeons may select different methods depending on their familiarity or experience with certain techniques. Thus, a meta-analysis based on recent studies was performed to systematically assess the effectiveness and safety of each tract dilation method.

\section{Materials and methods}

Study search and selection. The PubMed, EMBASE, Web of Science and Cochrane library databases were searched for relevant studies from database inception to 1 April 2019. Analysis was performed using the following MeSH key words: ('percutaneous nephrolithotomy', 'percutaneous lithotripsy', 
'PCNL', 'PNL' or 'PCN') AND ('one shot', 'single step', 'one stage', 'one-shot', 'single-step', 'one-stage', 'tract dilators', 'AD', 'MTD' or 'BD'). The search was restricted to RCTs published in English. The present study was prepared based on the Cochrane Handbook for Systematic Reviews of Interventions (16) and presented based on the Preferred Reporting Items for Systematic Reviews and Meta-analyses guidelines (17).

Inclusion and exclusion criteria. RCTs were selected for analysis according to the following inclusion criteria: i) RCTs that compared $\geq 2$ tract dilation techniques for PCNL; ii) RCTs that included patients aged $>18$ years; iii) the baseline characteristics were matched between groups; iv) RCTs were published in English and their full texts were available; v) at least one of the following types of data was available: Hemoglobin decrease, $\mathrm{X}$-ray exposure time, stone-free rate, operation time, length of hospital stay and blood transfusion rate. The exclusion criteria were as follows: i) RCTs that were not comparative studies; ii) the full texts were not accessible; and iii) RCTs that included patients aged $<18$ years.

Risk of bias assessments. The quality of the RCTs was assessed by two independent researchers using the Cochrane risk-of-bias criteria (16). Bias was evaluated based on the criteria of random sequence generation, allocation concealment, blinding of participants and personnel, blinding of outcome assessment, incomplete outcome data, selective reporting and other bias for each RCT, which allowed for grading of RCTs as low-risk, high-risk or unclear risk of bias. Providing the randomized sequence generation or allocation concealment indicated high risk of bias, the RCT was regarded as being of low quality, whereas if these factors were considered to be of low or unclear risk of bias, then the RCT would be regarded as being of high quality. In addition, intermediate risk of bias suggested that an RCT was of moderate quality.

Data extraction. The following data were extracted by two independent researchers (HLH and YCL): Name of first author, publication year, participant characteristics, stone burden, operational history, tract methods, hemoglobin decrease, $\mathrm{X}$-ray exposure time, stone-free rate, operation time, length of hospital stay, blood transfusion rate and complications. Disagreements were resolved by consensus. If any data were missing, attempts were made to contact the authors. The X-ray exposure time was the primary outcome, while the stone-free rate, blood transfusion rate, hemoglobin reduction and complications were denoted as the secondary outcomes.

Statistical analysis. The present study employed the Mantel-Haenszel statistical method to calculate risk ratios (RRs) and mean difference (MD) with $95 \%$ confidence intervals (CIs) for dichotomous data and continuous data to assess the overall outcomes of the four tract dilation methods for PCNL procedures. A $\chi^{2}$ test $(\mathrm{P}=0.05)$ and the $\mathrm{I}^{2}$ statistic were used to evaluate statistical heterogeneity among the studies by two independent researchers. $\mathrm{P}<0.05$ was considered to indicate a statistically significant difference and $\mathrm{I}^{2}>50 \%$ was considered to indicate heterogeneity. Fixed-effects and random-effect models were generated to analyze homogeneous and heterogeneous data, respectively. Sensitivity analysis was performed

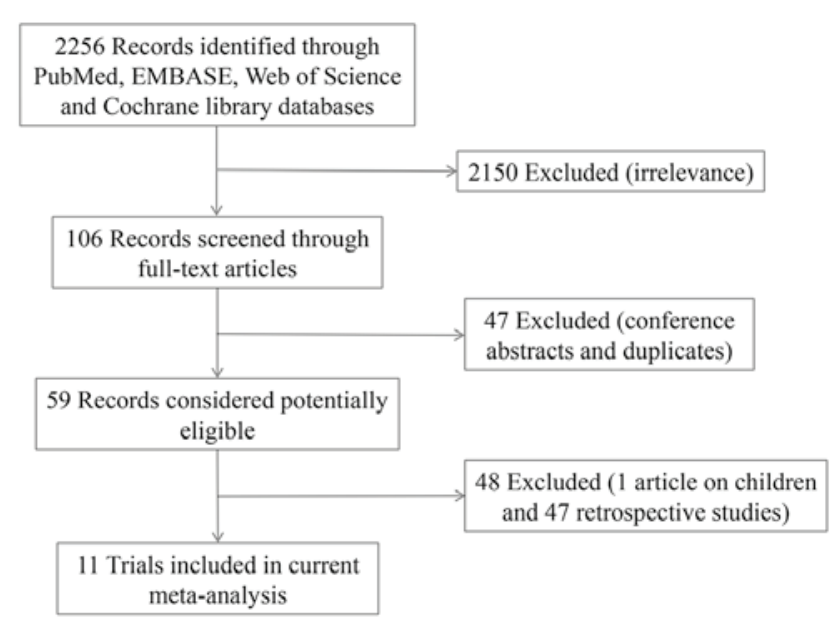

Figure 1. Search strategy and screening process of the literature performed in the present study.

by excluding trials with low quality or abnormal data. Possible publication bias was assessed by generating funnel plots for the studies. All meta-analyses were performed using RevMan version 5.2.

\section{Results}

Eligible studies and characteristics. A total of 110 potentially eligible reports were identified from the databases following screening of the titles and abstracts; 85 studies were excluded, as they were duplicates (37 articles) or retrospective trials (48 articles). In addition, 14 articles (13 conference abstracts and one study on pediatric patients) were excluded from the remaining 25 RCTs during the review of full-text articles based on the inclusion and exclusion criteria. Finally, 11 RCTs, comprising 1,415 cases, were included in the present meta-analysis (Fig. 1). Of note, six studies compared MTD with OSD $(8-12,14)$, one article compared MTD, OSD and BD (6); one compared MTD, OSD, BD and AD (13), two studies compared AD with OSD $(18,19)$, and one article compared MTD, BD and AD (20). The baseline characteristics and quality ratings of the studies included are presented in Table I.

Quality assessment and publication bias. Based on the Cochrane risk-of-bias criteria (16), two articles were graded as being of high quality and eight articles were graded as being of moderate quality, while one article was of low quality. The details of the quality assessment are presented in Fig. 2A and B. A lack of publication bias was demonstrated via the funnel plots for hemoglobin decrease, and the transfusion rate, successful dilation rate, one access rate and stone-free rate. The publication bias of the transfusion rate was also determined (Fig. 2C; data not shown for other items).

X-ray exposure time and access time. A total of eight studies compared MTD with OSD. The data were pooled for analysis with the random-effect model due to the significant heterogeneity among these studies $\left(\mathrm{P}<0.001 ; \mathrm{I}^{2}=97 \%\right)$. Significantly longer $\mathrm{X}$-ray exposure times for tract creation were determined for MTD compared with OSD (WMD, 


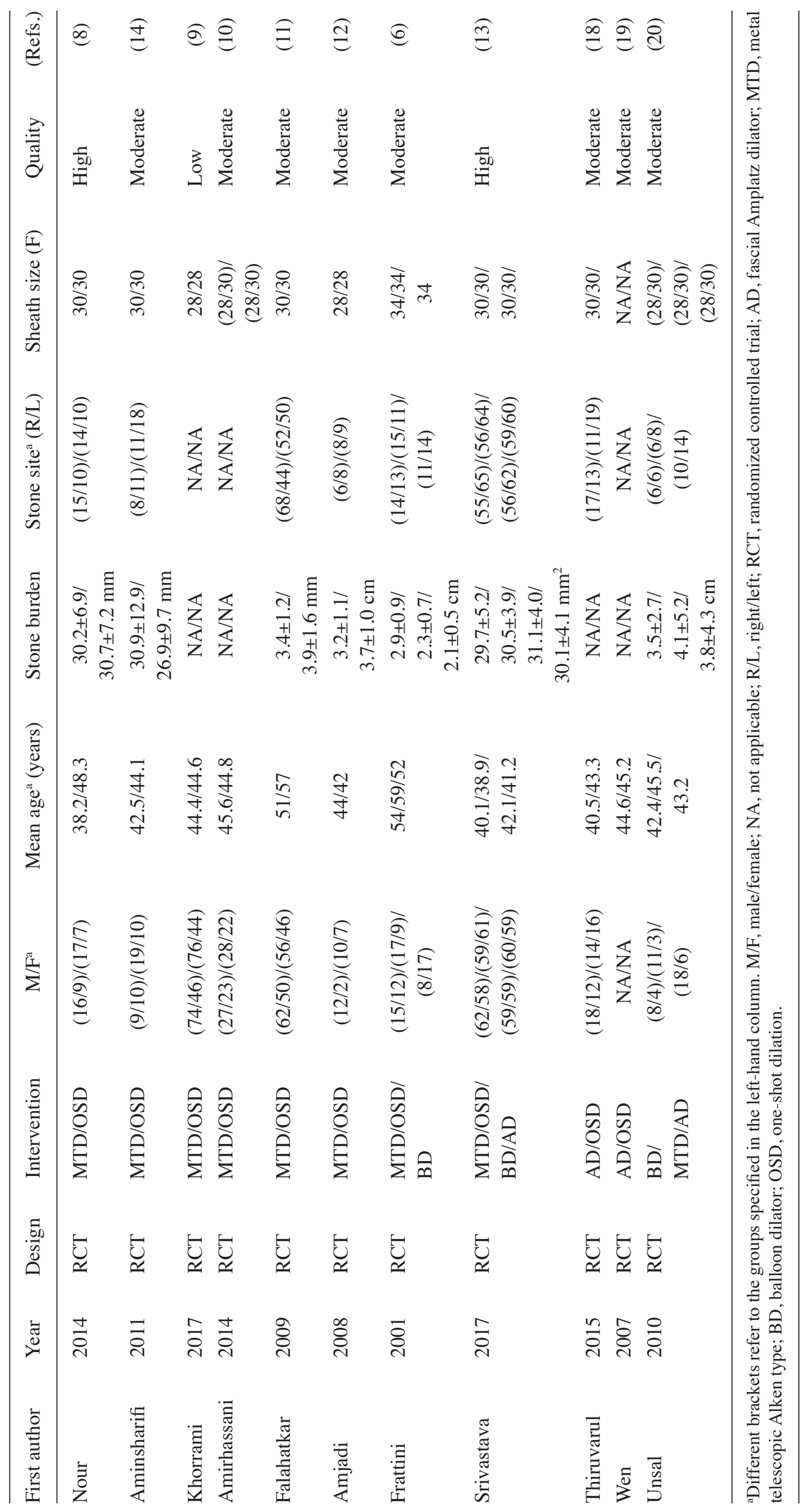



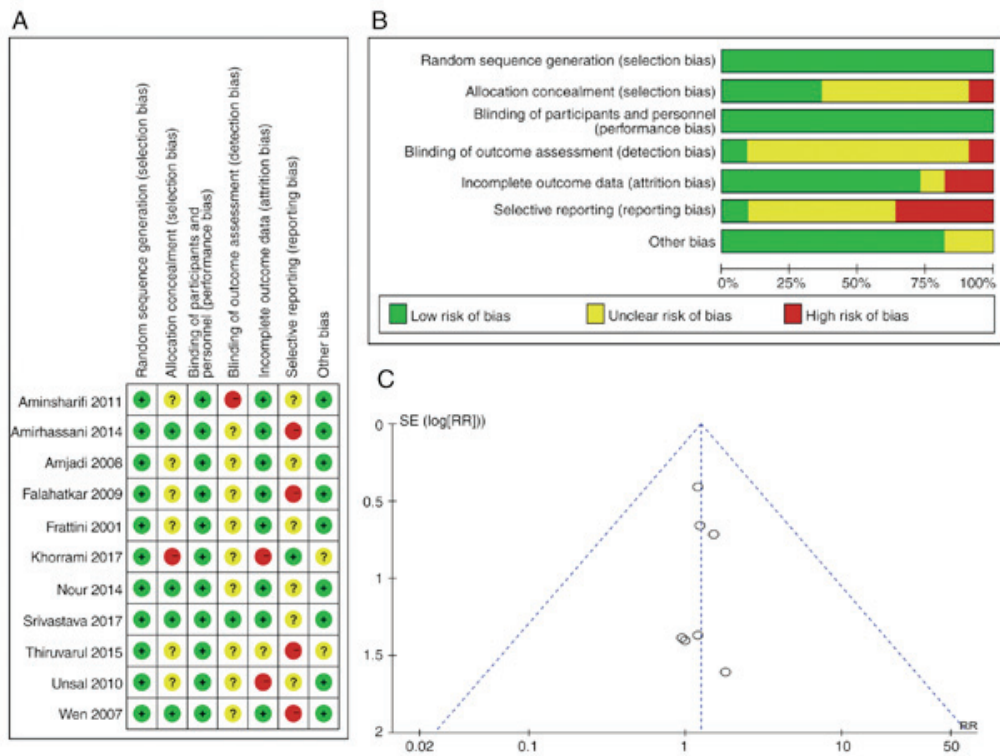

Figure 2. (A) Risk-of-bias summary for each study included. (B) Risk-of-bias graph for each study included. (C) Funnel plot for the transfusion rate. $\mathrm{SE}$, standard error; RR, risk ratio.

A X-ray exposure time between MTD and OSD

\begin{tabular}{|c|c|c|c|c|c|c|c|c|c|}
\hline Study or subgroup & Mean' & $\begin{array}{l}\text { MTD } \\
\text { SD }\end{array}$ & Total & Mean & $\begin{array}{l}O S D \\
\text { SD }\end{array}$ & Total & Weight & $\begin{array}{l}\text { Mean difference } \\
\text { IV, Random, } 95 \% \mathrm{CI}\end{array}$ & $\begin{array}{l}\text { Mean difference } \\
\text { IV, Random, } 95 \% \mathrm{CI}\end{array}$ \\
\hline Alireza Aminsharif 2011 & 67.2 & 40.8 & 19 & 46.2 & 24.6 & 29 & $11.6 \%$ & $21.00[0.59,41.41]$ & - \\
\hline Alok Sr & 62.1 & 13 & 120 & 36.8 & 7 & 120 & $20.1 \%$ & & \\
\hline Antonio Frattini 20 & 60 & & 27 & & 3 & 26 & $20.1 \%$ & 40.00 & - \\
\hline Hani H.Nour et al.2014 & 708 & 25.2 & 25 & 630 & 282 & 24 & $0.8 \%$ & $78.00[-35.25,191.25]$ & \\
\hline Moosen Amjadi 2008 & 81 & 53 & 14 & 27 & 15 & 17 & $8.2 \%$ & $54.00[25.34,82.66]$ & \\
\hline Shahriar Amirhassani 2014 & 48.4 & 15 & 50 & 41.2 & 17 & 50 & $19.0 \%$ & $7.20[0.92,13.48]$ & \\
\hline Siavash Falahatkar 2009 & 65 & 12 & 112 & 22 & 4 & 102 & $20.2 \%$ & $43.00[40.65,45.35]$ & - \\
\hline $95 \% \mathrm{Cl})$ & & & 367 & & & 3681 & $100.0 \%$ & $30.67[20.08,41.26]$ & $\bullet$ \\
\hline \multicolumn{9}{|c|}{$\begin{array}{l}\text { Heterogeneity: Tau' }=143.16 ; \mathrm{Ch}^{2}=186.93 \text {, df }=6(P<0.00001) ; 1^{2}=97 \% \\
\text { Test for overall effect: } Z=5.68(P<0.00001)\end{array}$} & $\begin{array}{llll}50 & 0 & 50 & 100 \\
\text { entall] } & \text { Favours [con }\end{array}$ \\
\hline
\end{tabular}

\section{B Access time between MTD and OSD}

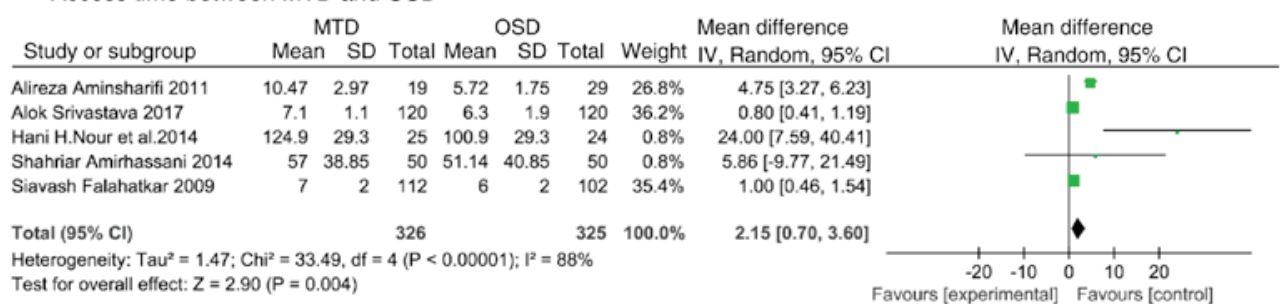

C X-ray exposure time between MTD and BD

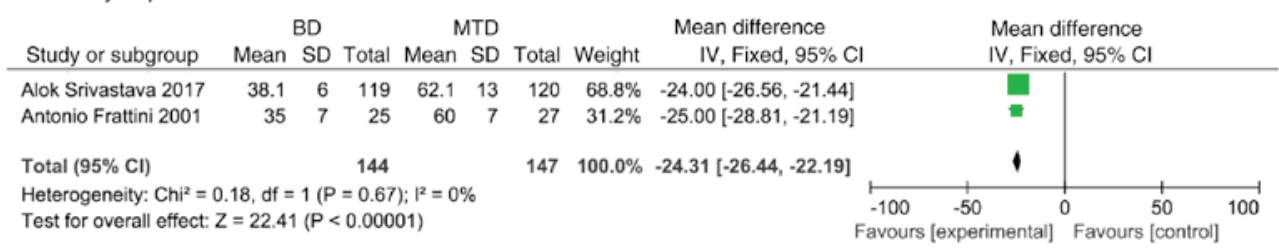

D X-ray exposure time between $\mathrm{BD}$ and $\mathrm{OSD}$

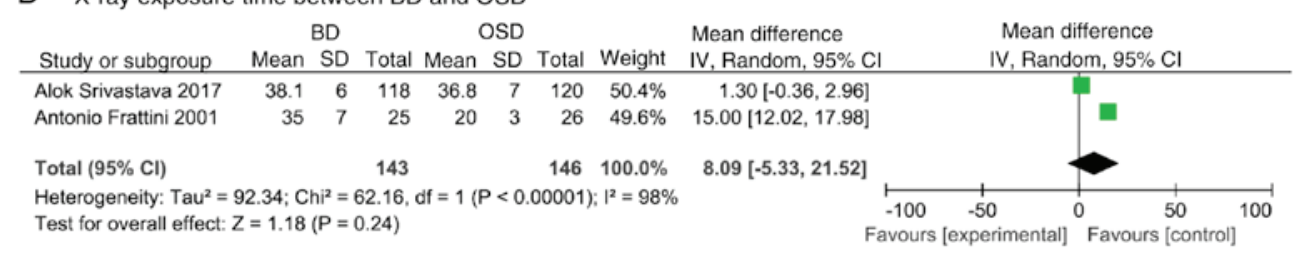

Figure 3. Forest plots for (A) X-ray exposure time compared between MTD and OSD, (B) access time compared between MTD and OSD, (C) X-ray exposure time compared between MTD and BD and (D) X-ray exposure time compared between BD and OSD. BD, balloon dilation; MTD, metal telescopic Alken type dilation; OSD, one-shot dilation; IV, inverse variance; SD, standard deviation; df, degrees of freedom. 


\section{A MTD vs OSD}

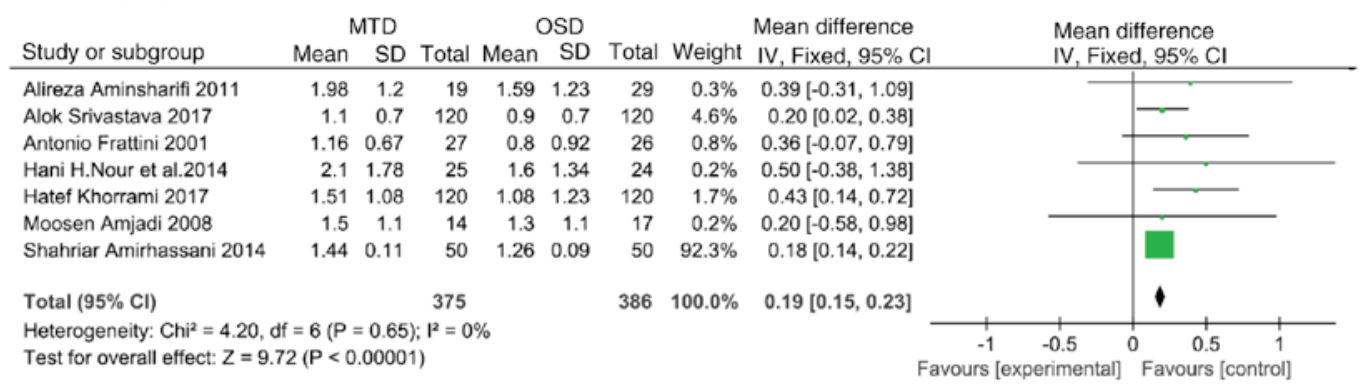

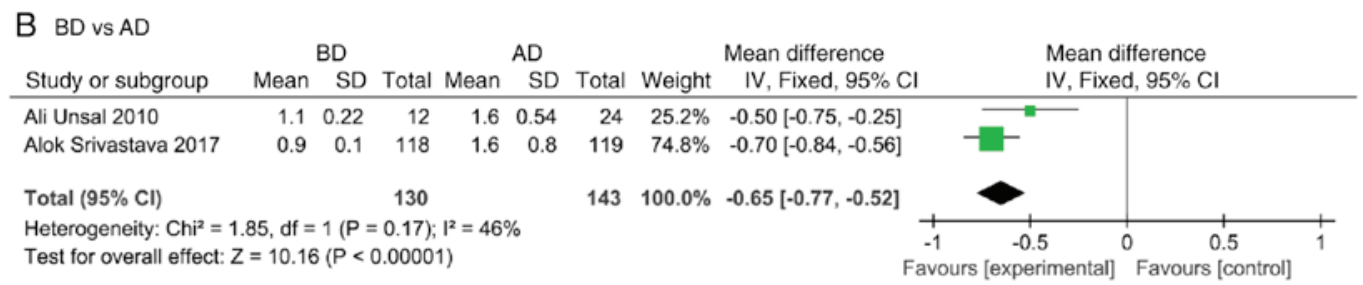

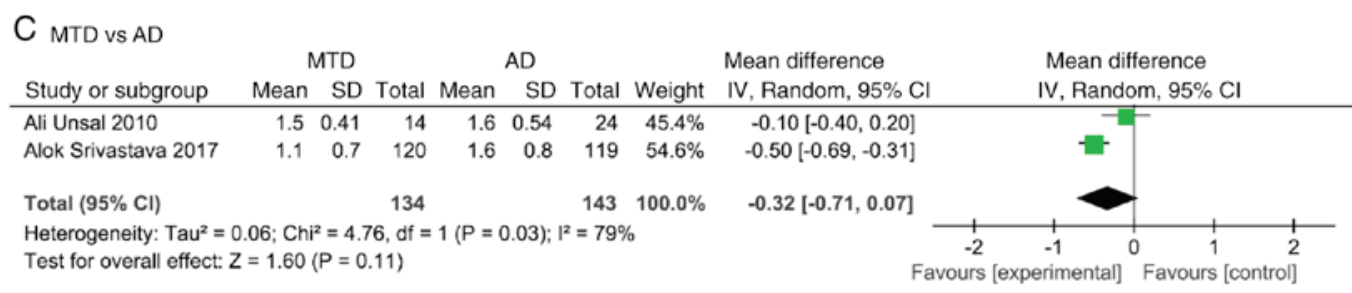

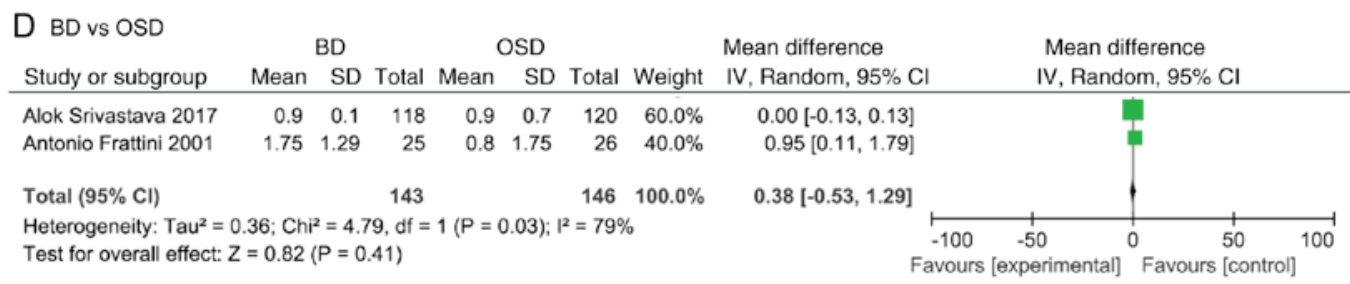

Figure 4. Forest plots for hemoglobin decrease compared among various treatments via meta-analysis. (A) MTD vs. OSD, (B) BD vs. AD, (C) MTD vs. AD and (D) BD vs. OSD. AD, fascial Amplatz dilation; BD, balloon dilation; MTD, metal telescopic Alken type dilation; OSD, one-shot dilation; IV, inverse variance; $\mathrm{SD}$, standard deviation; df, degrees of freedom.

30.67; 95\%CI, 20.08-41.26; P<0.001; Fig. 3A). Sensitivity analysis after exclusion of the trial by Nour et al (8) due to the inaccuracy of the definition of the X-ray exposure time and the access time compared to other studies revealed the same results [random-effects model; WMD, 30.27; 95\%CI, 19.63-40.92; $\mathrm{P}<0.001 ; \mathrm{I}^{2}=97 \%$ (result not shown)]. Similarly, for access time, heterogeneity was high $\left(\mathrm{P}<0.001 ; \mathrm{I}^{2}=88 \%\right)$, revealing that the access time for MTD was longer compared with that for OSD (random-effects model; WMD, 2.15; 95\%CI, 0.70-3.60; P<0.004; Fig. 3B). Following exclusion of the trial by Nour et al (8), the same results [random-effects model; WMD, 1.93; 95\%CI, 0.62-3.23; P<0.001; $\mathrm{I}^{2}=88 \%$ (result not shown)] were obtained. Heterogeneity of X-ray exposure times was low $\left(\mathrm{P}<0.67 ; \mathrm{I}^{2}=0 \%\right)$, revealing that the $\mathrm{X}$-ray exposure time for MTD was longer compared with that for OSD [fixed-effects model; WMD, -24.31; 95\%CI, -(26.44-22.19); $\mathrm{P}<0.001$; Fig. 3C], but the heterogeneity of $\mathrm{X}$-ray exposure time was high $\left(\mathrm{P}<0.001 ; \mathrm{I}^{2}=98 \%\right)$ between BD and OSD. Similar X-ray exposure time was observed between these groups (random-effects model; WMD, 8.09; 95\%CI, -5.33-21.52; P<0.24; Fig. 3D).
Hemoglobin decrease. A total of eight articles compared MTD with OSD. The data were pooled for analysis with fixed-effect models $\left(\mathrm{P}=0.65 ; \mathrm{I}^{2}=0 \%\right)$. A significant reduction in hemoglobin was determined for the MTD group compared with that in the OSD group (WMD, 0.19; 95\%CI, 0.15-0.23; P<0.001; Fig. 4A). In addition, two studies compared $\mathrm{BD}$ and $\mathrm{AD}$. The heterogeneity of hemoglobin decrease was low $\left(\mathrm{P}=0.17 ; \mathrm{I}^{2}=46 \%\right)$ and there was a significantly smaller decrease in hemoglobin in the $\mathrm{BD}$ group compared with that in the $\mathrm{AD}$ group [fixed-effects model; WMD, -0.65; 95\%CI, -(0.77-0.52); P<0.001; Fig. 4B]. The heterogeneity of hemoglobin decrease was high $(\mathrm{P}<0.03$; $\mathrm{I}^{2}=79 \%$ ) between MTD and AD. Similar hemoglobin decrease was determined between these groups (random-effects model; WMD, -0.32; 95\% CI, -0.71-0.07; P=0.11; Fig. 4C). The heterogeneity of hemoglobin decrease was high $\left(\mathrm{P}<0.03 ; \mathrm{I}^{2}=79 \%\right)$ and no statistically significant difference was determined in hemoglobin decrease between BD and OSD (random-effects model; WMD, 0.38; 95\%CI, -0.53-1.29; P=0.41; Fig. 4D).

Transfusion rate. The heterogeneity of transfusion rate between MTD and OSD $\left(\mathrm{P}=1.00 ; \mathrm{I}^{2}=0 \%\right), \mathrm{AD}$ and $\mathrm{OSD}(\mathrm{P}=0.83$; 
A Transfusion rate between MTD vs OSD

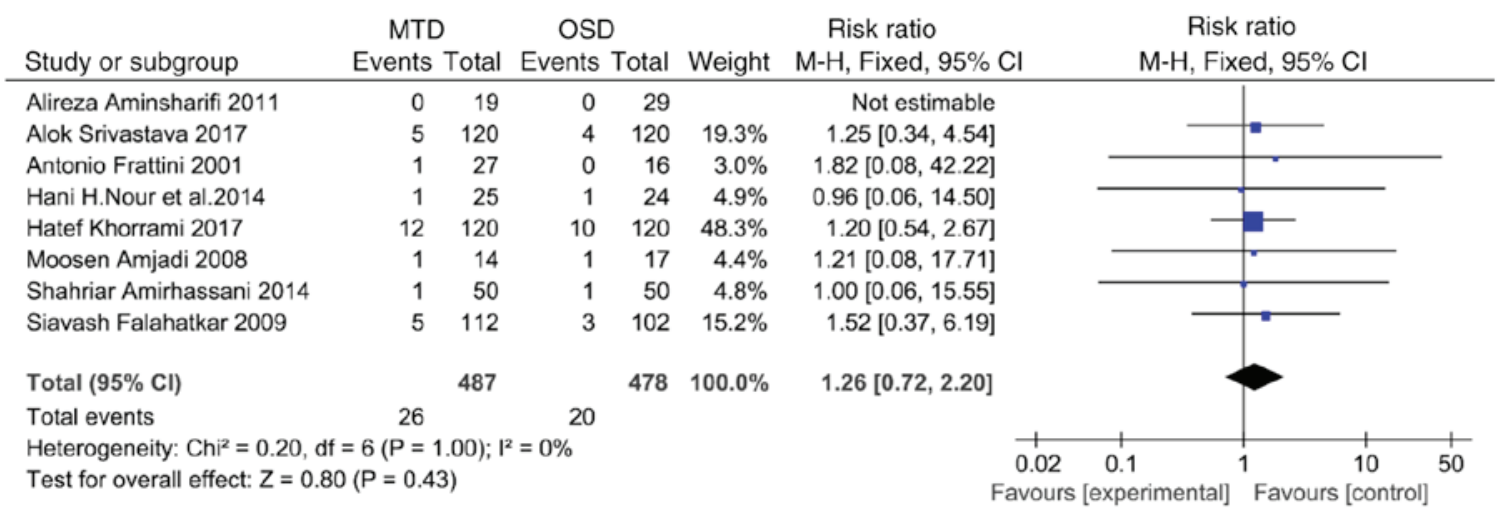

B Transfusion rate between $A D$ vs OSD

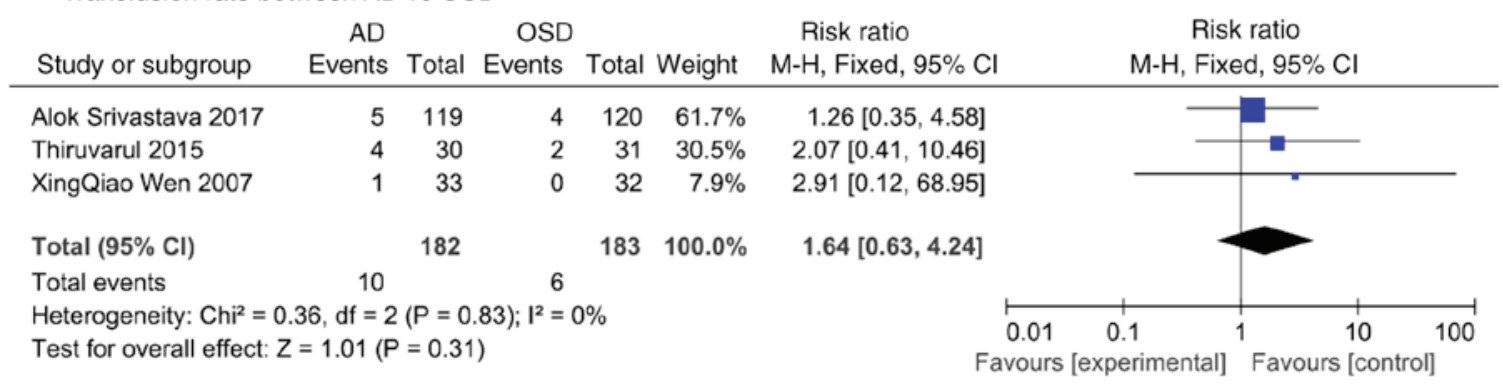

C Transfusion rate between $\mathrm{BD}$ vs OSD

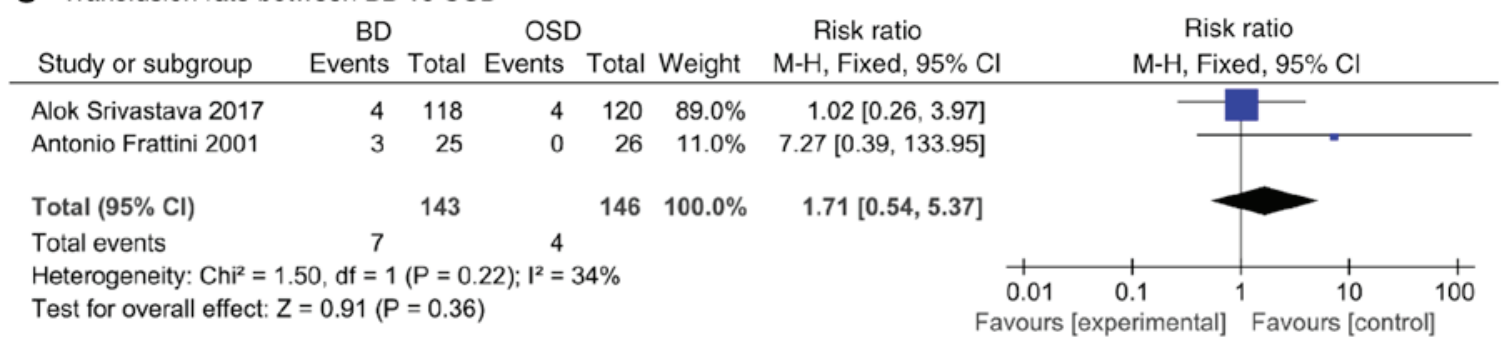

Figure 5. Forest plots for transfusion rate compared between (A) MTD and OSD, (B) AD and OSD and (C) BD and OSD. AD, fascial Amplatz dilation; BD, balloon dilation; MTD, metal telescopic Alken type dilation; OSD, one-shot dilation; M-H, Mantel-Haenszel; df, degrees of freedom.

$\left.\mathrm{I}^{2}=0 \%\right)$, and $\mathrm{BD}$ and $\mathrm{OSD}\left(\mathrm{P}=0.22 ; \mathrm{I}^{2}=34 \%\right)$ was low, revealing that MTD (fixed-effects model; RR, 1.26; 95\%CI, 0.72-2.20; $\mathrm{P}=0.43$; Fig. 5A), AD (fixed-effects model; RR, 1.64; 95\%CI, $0.63-4.24 ; \mathrm{P}=0.31$; Fig. 5B) and BD (fixed-effects model; RR, $1.71 ; 95 \% \mathrm{CI}, 0.54-5.37 ; \mathrm{P}=0.36$; Fig. 5C) had higher transfusion rates compared with OSD. The heterogeneity of transfusion rate between $\mathrm{BD}$ and MTD $\left(\mathrm{P}=0.46 ; \mathrm{I}^{2}=0 \%\right), \mathrm{BD}$ and $\mathrm{AD}(\mathrm{P}=0.65$; $\left.\mathrm{I}^{2}=0 \%\right)$, and MTD and $\mathrm{AD}\left(\mathrm{P}=0.91 ; \mathrm{I}^{2}=0 \%\right)$ was low. The transfusion rate was similar between BD and MTD (fixed-effects model; RR, 1.05; 95\%CI, 0.39-2.81; P=0.93; Fig. 6A), BD and $\mathrm{AD}$ (fixed-effects model; RR, 0.70; 95\%CI, 0.22-2.26; $\mathrm{P}=0.55$; Fig. 6B), and MTD and AD (fixed-effects model; RR, 0.96; 95\%CI, 0.33-2.81; P=0.94; Fig. 6C).

Successful dilation and one-access rates. The heterogeneity of successful dilation rate was low $\left(\mathrm{P}=0.80 ; \mathrm{I}^{2}=0 \%\right)$, revealing that MTD had a markedly higher successful dilation rate compared with OSD (fixed-effect model; RR, 1.02; 95\%CI, 1.00-1.04; $\mathrm{P}=0.09$; Fig. 6D); however, no statistical significance was obtained. The heterogeneity of one-access rate was low $\left(\mathrm{P}=0.49 ; \mathrm{I}^{2}=0 \%\right)$, and the one-access rate was similar between MTD and OSD (fixed-effects model; RR, 0.95; 95\%CI, 0.90-1.01; P=0.12; Fig. 7A).

Stone-free rate. The heterogeneity of stone-free rate between MTD and $\operatorname{OSD}\left(\mathrm{P}=0.58 ; \mathrm{I}^{2}=0 \%\right), \mathrm{BD}$ and $\mathrm{AD}\left(\mathrm{P}=0.95 ; \mathrm{I}^{2}=0 \%\right)$, and MTD and AD ( $\left.\mathrm{P}=0.77 ; \mathrm{I}^{2}=0 \%\right)$ was low. No statistically significant differences were observed in the stone-free rate between MTD and OSD (fixed-effects model; RR, 1.01; 95\% CI, 0.96-1.07; P=0.71; Fig. 7B), BD and AD (fixed-effects model; RR, 1.04; 95\%CI, 0.94-1.15; P=0.44; Fig. 7C), and MTD and AD (fixed-effects model; RR, 1.02; 95\% CI, 0.9-1.13; $\mathrm{P}=0.76 ; \mathrm{I}^{2}=0 \%$; Fig. 7D).

Hospital stay. A significant degree of heterogeneity between MTD and OSD was determined $\left(\mathrm{P}<0.001, \mathrm{I}^{2}=82 \%\right)$, and the data were pooled for analysis with a random-effects model. Similar lengths of hospital stay were determined between these groups (random-effects model; WMD, $-0.09 ; 95 \% \mathrm{CI}$, $-0.57-0.40 ;$ P $<0.72$; Fig. 8A). 
A Transfusion rate between BD vs MTD

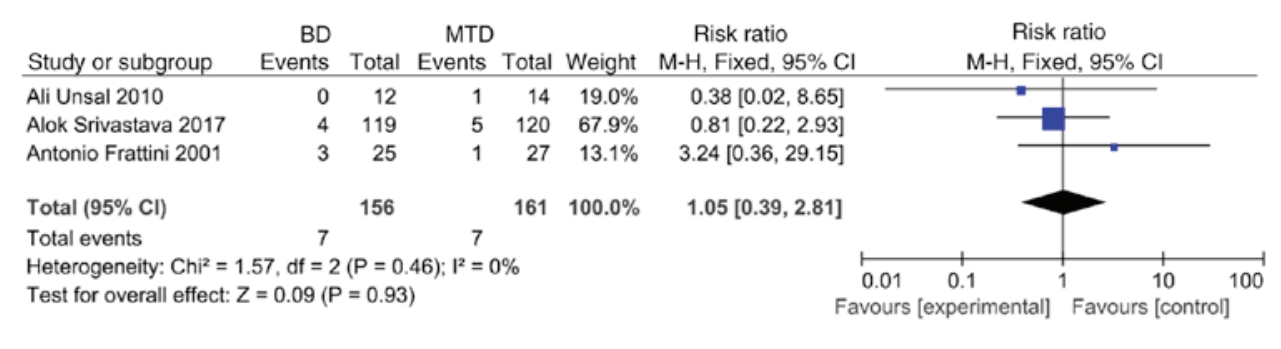

$B$ Transfusion rate between $B D$ vs $A D$

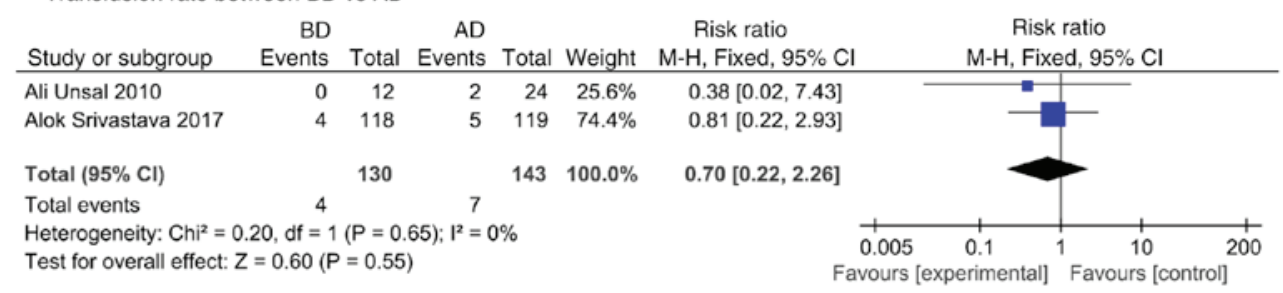

C Transfusion rate between MTD vs AD

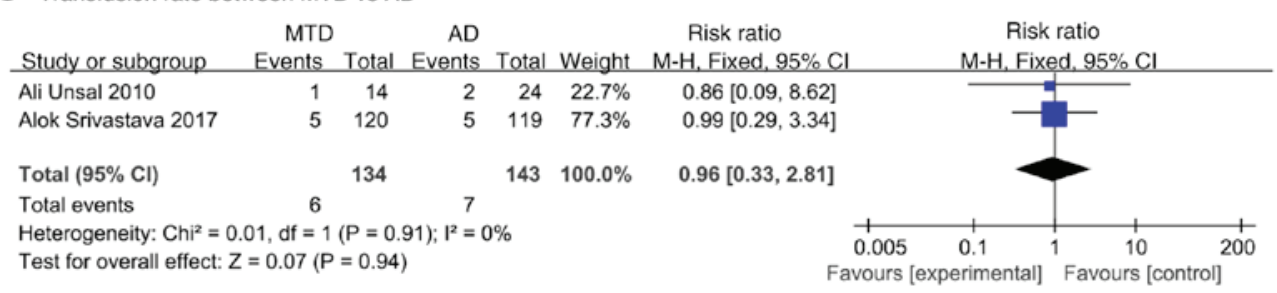

D Successful dilation rate between MTD vs OSD

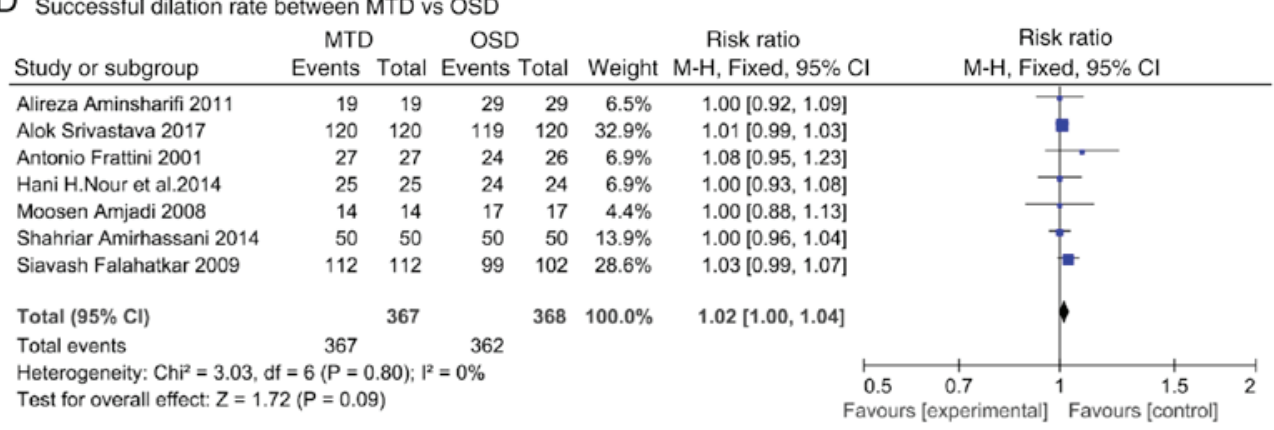

Figure 6. Forest plots for (A-C) transfusion rate compared between (A) BD and MTD, (B) BD and AD and (C) MTD and AD. (D) Successful dilation rate compared between MTD and OSD. AD, fascial Amplatz dilation; BD, balloon dilation; MTD, metal telescopic Alken type dilation; OSD, one-shot dilation; M-H, Mantel-Haenszel; df, degrees of freedom.

Collecting-system damage and hemorrhage rate. The heterogeneity of collecting system damage was low $\left(\mathrm{P}=0.36 ; \mathrm{I}^{2}=2 \%\right)$, no statistically significant differences were reported in damage to the collecting system between MTD and OSD (fixed-effects model; RR, 1.15; 95\%CI, 0.41-3.26; P=0.79; Fig. 8B). The heterogeneity of hemorrhage rate was low $\left(\mathrm{P}=0.83 ; \mathrm{I}^{2}=0 \%\right)$, revealing that a markedly increased hemorrhage rate was reported for MTD compared with OSD (fixed-effects model; RR, 1.95; 95CI\%, 0.50-7.57; P=0.33; Fig. 8C).

\section{Discussion}

Tract creation and dilation are fundamental steps in percutaneous renal surgery and are required for three traditional types of dilation, including MTD, AD and BD (21-23). OSD was first introduced by Frattini et al (6); several studies have investigated the safety and effectiveness of OSD compared with those of other methods (7-13). Numerous RCTs on these methods have been reported and a previous meta-analysis has been published by Cao et al (15). Of note, this previous meta-analysis included only four RCTs and analyzed three combinations of tract dilation methods without comparing the associated complications. Therefore, an integrated analysis of the four tract dilation techniques was required.

The present meta-analysis revealed that OSD was safe and effective for almost every adult patient, including those who previously underwent renal surgery. Significant differences were reported in X-ray exposure time and access time between MTD and OSD. Sensitivity analysis was performed by excluding a study that was abnormal due to inaccuracy of the definition of the X-ray exposure time and the access time, leading to marked differences in the data from other studies, and the same results were obtained. The hemoglobin decrease, transfusion rate and hemorrhage rate in the MTD and OSD 
A One access rate between MTD vs OSD

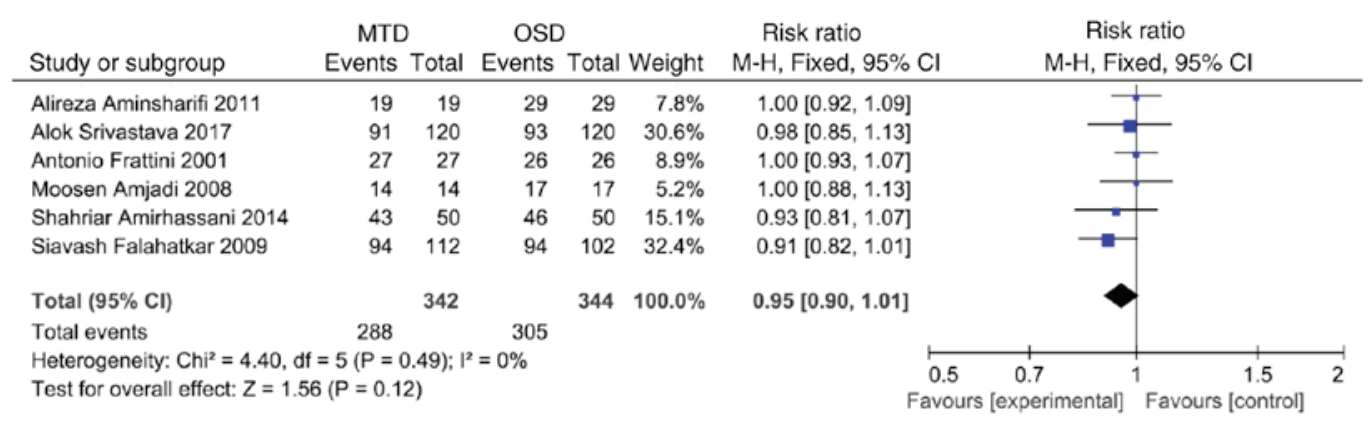

B Stone-free rate between MTD vs OSD

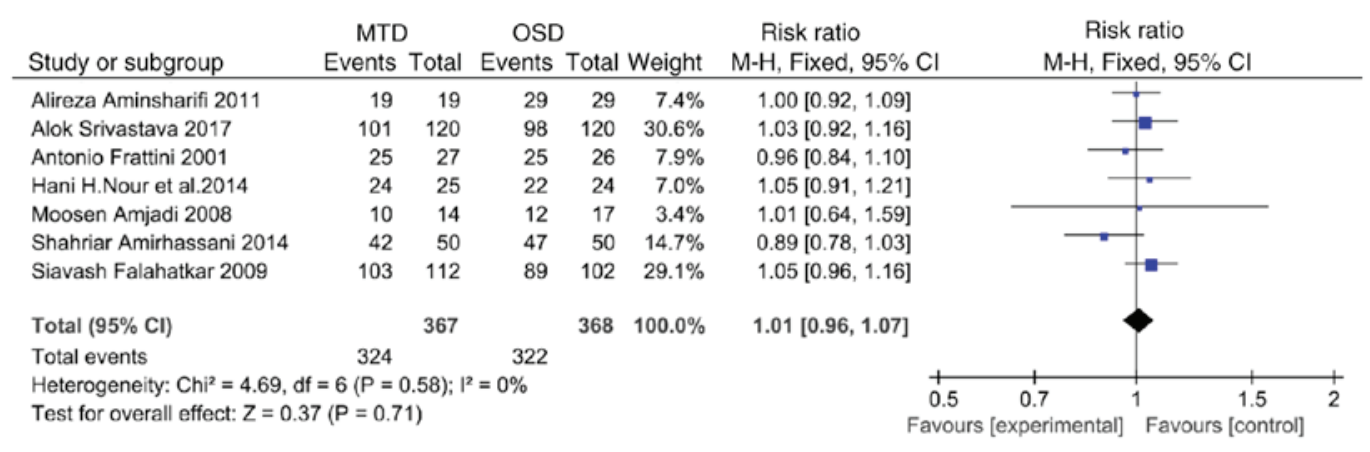

C Stone-free rate between $\mathrm{BD}$ vs $\mathrm{AD}$

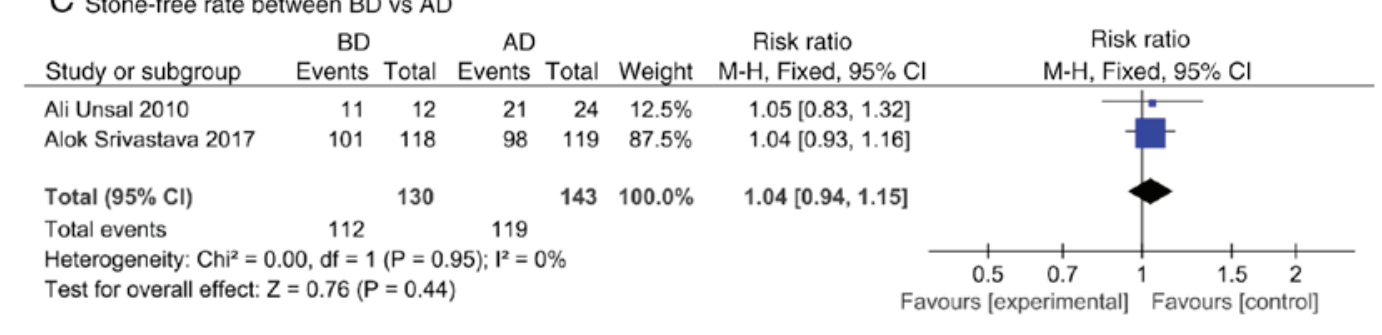

D Stone-free rate between MTD versus AD

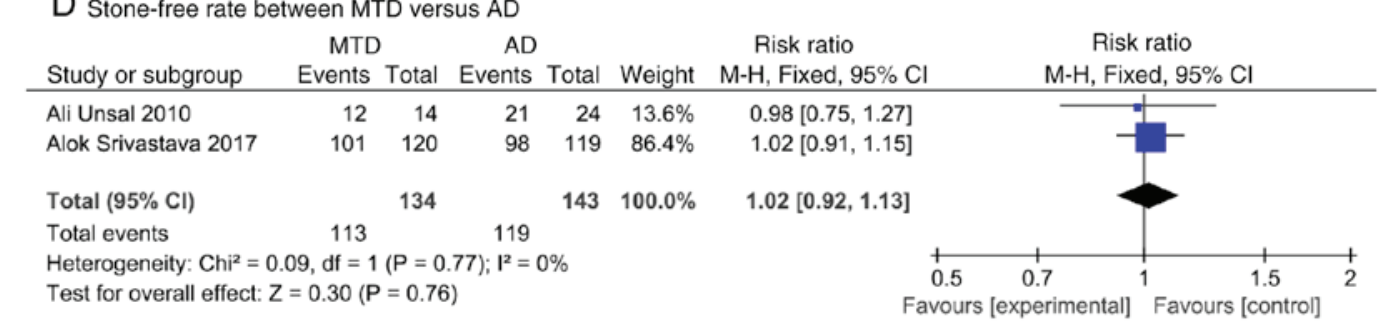

Figure 7. Forest plots for (A) one access rate compared between MTD and OSD and (B-D) stone-free rate compared between (B) MTD and OSD, (C) BD and AD and (D) MTD and AD. AD, Fascial Amplatz dilation; BD, balloon dilation; MTD, metal telescopic Alken type dilation; OSD, one-shot dilation; M-H, Mantel-Haenszel; df, degrees of freedom.

groups were also compared, as hemorrhage was characterized by blood drain within the nephrostomy tube, intermittent or continuous hematuria or gross hematuria with or without a decrease in hemoglobin and rarely required blood transfusion, and these three variables were linked but different. OSD was determined to significantly decrease the transfusion rate, hemorrhage rate and the extent of hemoglobin decrease compared with those of MTD. These results support the results of previous studies $(6,8-14,18-20)$. In addition, no statistically significant differences were observed between the two groups regarding the stone-free rate. By contrast, Falahatkar et al (11) and Srivastava et al (13) reported that OSD required more auxiliary procedures. This may be due to the higher proportion of complex stones in the OSD; it may also indicate that OSD has limited efficacy in managing complex stones. The present meta-analysis revealed that, as compared with MTD, OSD was associated with a lower rate of complications, including damage to the collecting system and hemorrhage. These results indicated that the OSD technique may be widely used; however, Srivastava et al (13) reported on two patients with minor pelvic perforations and injuries to the collecting system. These patients had a similar history of ipsilateral open surgery. OSD benefits from increased radial force and reduced axial force, yet the high resistance of fascial dilation requires more radial force, which may be the cause of complications. In addition, several studies reported on a number of unsuccessful procedures in patients who underwent open surgery $(6,11,13)$, whereas 
A Hospital stay

\begin{tabular}{|c|c|c|c|c|c|c|c|c|c|}
\hline \multirow{2}{*}{ Study or subgroup } & \multicolumn{2}{|c|}{ MTD } & \multicolumn{4}{|c|}{ OSD } & \multirow{2}{*}{\multicolumn{2}{|c|}{$\begin{array}{c}\text { Mean difference } \\
\text { Weight IV, Random, } 95 \% \mathrm{Cl}\end{array}$}} & \multirow{2}{*}{$\begin{array}{c}\text { Mean difference } \\
\text { IV, Random, } 95 \% \mathrm{Cl}\end{array}$} \\
\hline & Mean & SD T & Total & Mean & SDT & Total & & & \\
\hline Alok Srivastava 2017 & 3.1 & 1.6 & 120 & 2.7 & 2.1 & 120 & $24.4 \%$ & $0.40[-0.07,0.87]$ & \\
\hline Antonio Frattini 2001 & 4 & 1 & 27 & 5 & 1 & 26 & $22.8 \%$ & $-1.00[-1.54,-0.46]$ & $\varpi$ \\
\hline Hani H.Nour et al.2014 & 4.6 & 1 & 25 & 4.3 & 1 & 24 & $22.3 \%$ & $0.30[-0.26,0.86]$ & \\
\hline Hatef Khorrami 2017 & 2.28 & 0.61 & 120 & 2.36 & 0.67 & 120 & $30.6 \%$ & $-0.08[-0.24,0.08]$ & \\
\hline Total $(95 \% \mathrm{Cl})$ & & & 292 & & & 290 & $100.0 \%$ & $-0.09[-0.57,0.40]$ & \\
\hline $\begin{array}{l}\text { Heterogeneity: } \text { Tau }^{2}=0 \text {. } \\
\text { Test for overall effect: } Z\end{array}$ & & & & & & & & & $\begin{array}{lccc}-2 & -1 & 0 & 1 \\
\text { perimental] } & \text { Favours }\end{array}$ \\
\hline
\end{tabular}

B

Collecting system damage

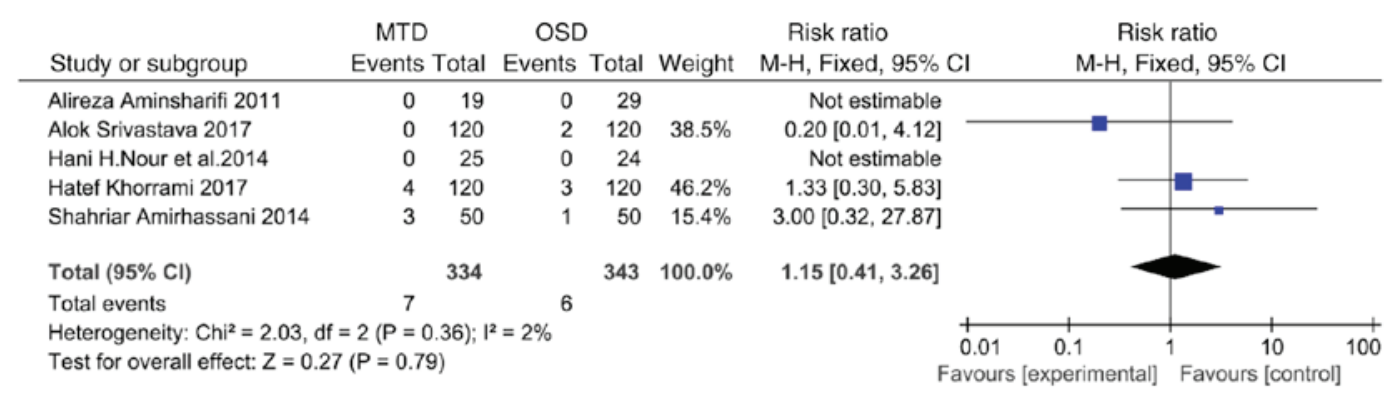

C hemorrhage rate

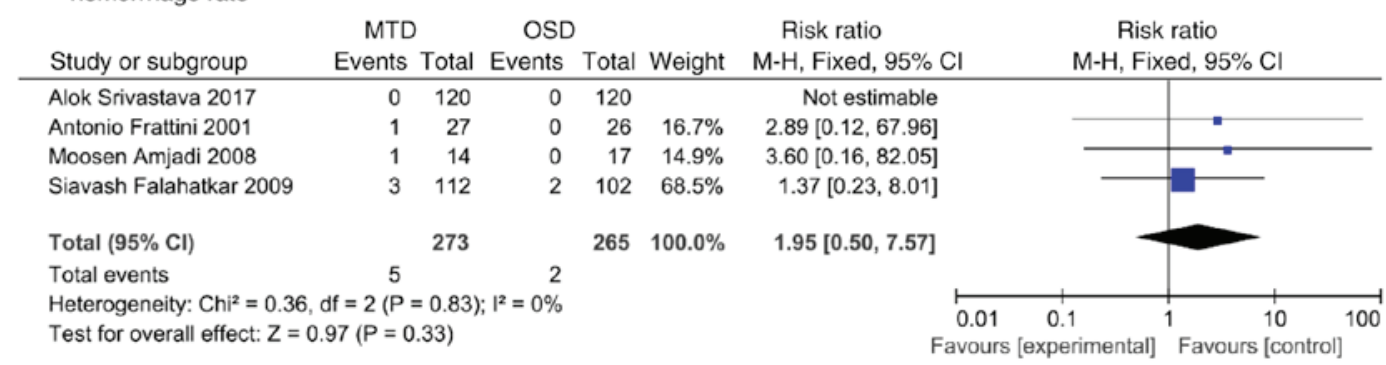

Figure 8. Forest plots for comparison of adverse events between MTD and OSD via meta-analysis. (A) Hospital stay, (B) collecting system damage and (C) hemorrhage rate. MTD, metal telescopic Alken type dilation; OSD, one-shot dilation IV, inverse variance; M-H, Mantel-Haenszel; SD, standard deviation; df, degrees of freedom.

OSD was determined to be as effective as MTD in previously operated patients $(10,12,24,25)$. Frattini et al (6) and Falahatkar et al (11) suggested that the high resistance of perirenal scar tissue due to previous kidney surgery, which prevented fascial dilator passage, or renal supermotility and rotation during dilation, may lead to avulsion of the entire organ. In the present meta-analysis, the rates of successful dilation and one access were compared. OSD was determined to have a markedly lower successful dilation rate. Advancements in equipment and the experience of surgeons in employing various techniques may also account for the differences determined. In addition, Aminsharifi et al (14) demonstrated that OSD caused more parenchymal damage than MTD. The difference between the two methods, an insufficient number of patients and inadequate follow-up time may have influenced the results. Furthermore, it is uncertain whether the formation of small new scars affects renal function. Lee and Stoller (26) suggested that the differences reported may be explained by the surgeon's familiarity and experience rather than the dilator systems. The hemoglobin decrease associated with MTD was previously determined to not be significantly different compared with that resulting from AD (13,27); however, in the present study, MTD was reported to lead to a lower hemoglobin decrease compared with AD. This could be due to trauma as a result of the cycle of insertion and removal of the dilator in and out of the tract during sequential exchange, increasing the risk of bleeding. However, the transfusion rate and stone-free rate were similar between the two groups. AD and MTD are used to create the tract by using axial and radial forces, which have a higher chance of successful tract creation compared with OSD. The spinning movement of the sheath during tract creation serves an important role in decreasing the risk of kidney or guidewire shift (28). Thus, these methods, particularly $\mathrm{AD}$, are frequently used in patients who have previously undergone kidney surgery. As of the small tip of these dilators, it is easy to guide along smaller tracts from previous dilations, regardless of the density of scar tissue (25). A number of studies reported that BD had reduced X-ray exposure time and hemoglobin decrease compared with $\mathrm{AD}$ and MTD $(13,20,29)$, as the inflated balloon provides constant pressure and tamponades the small injured vessels. In addition, $\mathrm{BD}$ was previously proposed to be more likely to fail in patients who underwent kidney surgery $(13,30)$. This may be due to the low axial force in $\mathrm{BD}$ and the lack of constant dilation. Kijvikai and de la Rosette (31) reported that BD was not suitable for complete staghorn calculus. The space between these stones and the collecting system may be inadequate and the tapered end of the dilator may create a small tract into the collecting system or split the calix. 
Of note, the present study has certain limitations. A variety of factors, including the stone burden, body mass index, hydronephrosis grade or the number of previous surgeries may have influenced the results; subgroup analyses were not performed due to limitations of the studies included. In addition, other complications were not compared, including the presence of pleural effusion, post-operative urinary tract infections or post-operative fever due to a lack of data. Furthermore, the heterogeneity of the data may have affected the results of X-ray exposure time and access time; the high heterogeneity may have been due to the different definitions among the studies included. Despite these limitations, there are certain advantages of the present study regarding the quality and quantity of articles analyzed, and the results of comparisons with previous meta-analyses in terms of both quality and quantity (15), as results from the present study were more comprehensive.

In the present meta-analysis, OSD was determined to be a safer method in almost every adult patient, including those who underwent renal surgery previously, compared to $\mathrm{AD}$ and MTD; however, OSD has a considerable risk of serious complications if the surgeon is inexperienced. Therefore, the present study proposed that surgeons with technical expertise in OSD should perform this procedure. BD was also determined to be more effective and safer than AD and MTD in patients that had not undergone renal surgery previously. By contrast, $\mathrm{BD}$ is an expensive procedure and unsuitable for patients with a history of renal surgery. For patients who have undergone kidney surgery, AD and MTD are safer methods of dilation. However, additional RCTs are required to determine the best method for PCNL.

\section{Acknowledgements}

Contents of this study were previously presented as a poster at the 37th World Congress of Endourology (1st November 2019; Abu Dhabi, United Arab Emirates; abstract no. MP23-04).

\section{Funding}

No funding was received.

\section{Availability of data and materials}

The datasets used and/or analyzed during the present study are available from the corresponding author on reasonable request.

\section{Authors' contributions}

YW performed the statistical analysis and wrote the manuscript. YX and HLH performed the literature search. HLH and YCL extracted the data. BLQ performed the quality assessment. YW and YX performed the meta-analysis and interpreted the results. SGW was a major contributor in conceiving and designing the manuscript. SGW also calibrated the final version and revised the manuscript. All authors read and approved the final manuscript.

\section{Ethics approval and consent to participate}

Not applicable.

\section{Patient consent for publication}

Not applicable.

\section{Competing interests}

The authors declare that they have no competing interests.

\section{References}

1. Türk C, Petř́k A, Sarica K, Seitz C, Skolarikos A, Straub M and Knoll T: EAU guidelines on diagnosis and conservative management of urolithiasis. Eur Urol 69: 468-474, 2016.

2. Sivalingam S, Al-Essawi T and Hosking D: Percutaneous nephrolithotomy with retrograde nephrostomy access: A forgotten technique revisited. J Urol 189: 1753-1756, 2013.

3. Handa RK, Matlaga BR, Connors BA, Ying J, Paterson RF, Kuo RL, Kim SC, Lingeman JE, Evan AP and Willis LR: Acute effects of percutaneous tract dilation on renal function and structure. J Endourol 20: 1030-1040, 2006.

4. Stoller ML, Wolf JS Jr and St Lezin MA: Estimated blood loss and transfusion rates associated with percutaneous nephrolithotomy. J Urol 152: 1977-1981, 1994.

5. Davidoff $\mathrm{R}$ and Bellman GC: Influence of technique of percutaneous tract creation on incidence of renal hemorrhage. J Urol 157: 1229-1231, 1997.

6. Frattini A, Barbieri A, Salai P, Sebastio N, Ferretti S, Bergamaschi E and Cortellini P: One shot: A novel method to dilate the nephrostomy access for percutaneous lithotripsy. J Endourol 15: 919-923, 2001.

7. Rusnak B, Castañeda-Zuñiga W, Kotula F, Herrera M and Amplatz K: An improved dilator system for percutaneousnephrostomies. Radiology 144: 174, 1982.

8. Nour HH, Kamal AM, Zayed AS, Refaat H, Badawy MH and El-Leithy TR: Single-step renal dilatation in percutaneous nephrolithotomy: A prospective randomised study. Arab J Urol 12: 219-222. 2014.

9. Khorrami MH, Izadpanahi MH, Mohammadi M, Alizadeh F, Zargham M, Khorrami F and Isfahani FF: Comparison of two treatment methods 'one shot' and 'sequential' on reduction the level of hemoglobin in patients with percutaneous nephrolithotripsy in Al Zahra hospital in 2012-2013. Adv Biomed Res 6: 84, 2017.

10. Amirhassani S, Mousavi-Bahar SH, Iloon Kashkouli A and Torabian S: Comparison of the safety and efficacy of one-shot and telescopic metal dilatation in percutaneous nephrolithotomy: A randomized controlled trial. Urolithiasis 42: 269-273, 2014.

11. Falahatkar S, Neiroomand H, Akbarpour M, Emadi SA and Khaki N: One-shot versus metal telescopic dilation technique for tract creation in percutaneous nephrolithotomy: Comparison of safety and efficacy. J Endourol 23: 615-618, 2009.

12. Amjadi M,Zolfaghari A,Elahian A and Tavoosi A: Percutaneous nephrolithotomy in patients with previous open nephrolithotomy: One-shot versus telescopic technique for tract dilatation. J Endourol 22: 423-425, 2008.

13. Srivastava A, Singh S, Dhayal IR and Rai P: A prospective randomized studycomparing the four tract dilation methods of percutaneous nephrolithotomy. World J Urol 35: 803-807, 2017.

14. Aminsharifi A, Alavi M, Sadeghi G, Shakeri S and Afsar F: Renal parenchymal damage after percutaneous nephrolithotomy with one-stage tract dilation technique: A randomized clinical trial. J Endourol 25: 927-931, 2011.

15. Cao DH, Liu LR, Liu HW and Wei Q: A comparison among four tract dilation methods of percutaneous nephrolithotomy: A systematic review and meta-analysis. Urolithiasis 41: 523-530, 2013.

16. Higgins JPT, Green S (eds). Cochrane Handbook for Systematic Reviews of Interventions. Version 5.1.0 (updated March 2011). The Cochrane Collaboration, 2011. http://www.cochranehandbook.org. Accessed November 22, 2017. 
17. Liberati A, Altman DG, Tetzlaff J, Mulrow C, Gøtzsche PC, Ioannidis JP, Clarke M, Devereaux PJ, Kleijnen J and Moher D: The PRISMA statement for reporting systematic reviews and meta-analyses of studies that evaluate healthcare interventions: Explanation and elaboration. BMJ 339: b2700, 2009.

18. Thiruvarul PV, Periasamy P and Pitchai BK: Single step vs serial dilatation for tract creation in percutaneous nephrolithotomy: A randomized controlled trial. J Evolution Med Dental Sci 91 $15669-15672,2015$.

19. Wen X, Gao X, Li X, Lu M, Cai Y, Qiu J and Xiao C: One-step percutaneous nephrostomy in patients with a history of open nephrolithotomy: Comparison with the fascial dilator system. J Endourol 21: 1281-1285, 2007.

20. Unsal A, Koca G, Reşorlu B, Bayindir M and Korkmaz M: Effect of percutaneous nephrolithotomy and tract dilatation methods on renal function: Assessment by quantitative single-photon emission computed tomography of technetium-99m-dimercaptosuccinic acid uptake by the kidneys. J Endourol 24: 1497-1502, 2010.

21. Alken P, Hutschenreiter G and Günther R: Percutaneous kidney stone removal. Eur Urol 8: 304-311, 1982.

22. Kessaris DN, Bellman GC, Pardalidis NP and Smith AG: Management of hemorrhage after percutaneous renal surgery. J Urol 153: 604-608, 1995.

23. Roth RA and Beckmann CF: Complications of extracorporeal shockwave lithotripsy and percutaneous nephrolithotomy. Urol Clin North Am 15: 155-166, 1988.

24. Ziaee SA, Karami H, Aminsharifi A, Mehrabi S, Zand S and Javaherforooshzadeh A: One-stage tract dilation for percutaneous nephrolithotomy: Is it justified? J Endourol 21: 1415-1420, 2007.
25. Lojanapiwat B: Previous open nephrolithotomy: Does it affect percutaneous nephrolithotomy techniques and outcome? J Endourol 20: 17-20, 2006

26. Lee KL and Stoller ML: Minimizing and managing bleeding after percutaneous nephrolithotomy. Curr Opin Urol 17: 120-124, 2007.

27. Ozok HU, Sagnak L, Senturk AB, Karakoyunlu N, Topaloglu H and Ersoy H: A comparison of metal telescopic dilators and Amplatz dilators for nephrostomy tract dilation in percutaneous nephrolithotomy. J Endourol 26: 630-634, 2012.

28. McDougall EM, Liatsikos EN, Dinlenc CZ, et al: Percutaneous approaches to the upper urinary tract. In: Walsh PC, Vaughan ED, Wein AJ,Retik AB, eds. Campbell's Urology. 8th ed. Philadelphia: WB Saunders. pp 3320-3369. 2002.

29. Safak M, Gögüs C and Soygür T: Nephrostomy tract dilation using a balloon dilator in percutaneous renal surgery: Experience with 95 cases and comparison with the fascial dilator system. Urol Int 71: 382-384, 2003

30. Joel AB, Rubenstein JN, Hsieh MH, Chi T, Meng MV and Stoller ML: Failed percutaneous balloon dilation for renal access: Incidence and risk factors. Urology 66: 29-32, 2005.

31. Kijvikai K and de la Rosette J: Surgical challenges of percutaneous nephrolithotomy. Business Briefing: European Kidney \& Urological Disease. 82-84. 2006.

(i) (9) This work is licensed under a Creative Commons Attribution-NonCommercial-NoDerivatives 4.0 International (CC BY-NC-ND 4.0) License. 